\title{
Biodiversité et structure des communautés de poissons du lac Hlan au Bénin
}

\author{
Elie MONTCHOWUI ${ }^{1 *}$, Antoine CHIKOU ${ }^{1}$, Marie-José KOGBETO ${ }^{2}$ et Philippe \\ LALEYE $^{1}$ \\ ${ }^{1}$ Laboratoire d'Hydrobiologie et d'Aquaculture, Faculté des Sciences Agronomiques, Université d'Abomey- \\ Calavi, 01 BP 526 Cotonou, Bénin. \\ ${ }^{2}$ CERPA Mono-Couffo, Secteur Comè, BP 31 Lokossa, Bénin. \\ *Auteur correspondant, Courriel: e.montchowui@yahoo.fr
}

\section{RÉSUMÉ}

Cette étude concerne l'un des petits lacs du sud Bénin : le lac Hlan. Elle vise à faire l'inventaire de l'ichtyofaune et à déterminer l'organisation des peuplements de poissons du lac. L'ichtyofaune a été échantillonnée mensuellement de septembre 2004 à août 2005 au moyen de la pêche artisanale. Les données d'inventaire de la pêche artisanale sont complétées par celles des pêches expérimentales avec la senne à très fine maille (filet moustiquaire) utilisée dans les zones peu profondes. Les peuplements de poissons ont été étudiés au moyen de descripteurs populationnels (la richesse spécifique, la densité, la biomasse et les indices de diversité) et d'analyses statistiques. 37 espèces de poisson, réparties en 29 genres et 18 familles, ont été recensées. Les familles les plus représentées sont les cichlidés ( 8 espèces), les mormyridés (4 espèces) et les cyprinidés (3 espèces). L'organisation de l'assemblage conserve toujours la même structure générale, à laquelle environ 10 espèces ont principalement participé. Trois espèces sur les 10 représentent $80 \%$ de l'abondance des captures commerciales. Ce sont Heterotis niloticus, Sarotherodon galilaeus et Tilapia zillii. La majorité des espèces participant aux captures de la pêche artisanale sont des espèces ubiquistes occupant tous les endroits du lac où la nourriture est disponible.

(C) 2008 International Formulae Group. All rights reserved.

Mots clés: Biodiversité, Ichtyofaune, Assemblage, Lac Hlan, Bénin.

\section{INTRODUCTION}

La surexploitation des ressources en poissons des lacs et lagunes est aujourd'hui largement reconnue à l'échelle mondiale comme au niveau africain ou national. Elle se traduit en particulier par une situation de forte régression des captures de jour en jour malgré l'accroissement des performances des engins de pêche utilisés (Lalèyè, 1995 ; Levêque et Paugy, 1999).

Cette situation a conduit à des mesures de conservation et de gestion rationnelle des stocks des peuplements de poissons et des pêcheries. Ces mesures reposent sur des informations précises en ce qui concerne l'écologie, la composition de la faune ichtyologique et son niveau d'exploitation dans les plans et cours d'eau. En effet, sans une connaissance approfondie de ces paramètres, ces mesures sont d'avance vouées à l'échec.

$\mathrm{Au}$ Bénin, l'ichtyofaune des eaux continentales est peu documentée. Les quelques études récentes disponibles (Adite et Rudi, 1995 ; Lalèyè et al., 2003 ; Lalèyè et al., 2004) ne sont consacrées qu'aux grands plans et cours d'eau. Il en existe d'autres, non moins négligeables, du point de vue de leur contribution à la production halieutique nationale. C'est le cas du lac Hlan, dans le Sud-Bénin. Très peu de travaux ont été réalisés sur cet écosystème pour mieux comprendre son fonctionnement notamment dans un but de gestion et, sur sa faune piscicole pour une meilleure connaissance de ses ressources dans le but d'une conservation 
et d'une utilisation rationnelle. Le présent travail vise à caractériser l'ichtyofaune du lac Hlan et à définir les variations temporelles de ses peuplements de poissons.

\section{MATÉRIEL ET MÉTHODES}

\section{Milieu d'étude}

Le lac Hlan (Figure 1) est situé dans le village de Kpomè dans le département de l'Atlantique à $80 \mathrm{~km}$ de la côte Atlantique entre 6 $6^{\circ} 56^{\prime} 35,05^{\prime \prime}$ 'de latitude Nord et $2^{\circ} 19^{\prime} 57,32^{\prime \prime}$ de longitude Est. Il est situé dans le bassin versant de la rivière Hlan et est alimenté en eau par des petits cours d'eau dont : Samion (15 km), Da (16 km), Hoho (17 $\mathrm{km})$, Hlan $(32,8 \mathrm{~km})$ et Koto $(10 \mathrm{~km})$. Le lac est relié au fleuve Ouémé et la rivière Sô par l'intermédiaire des marécages dans lesquels ces cours d'eau déversent pendant la crue leurs eaux, qui permettent des migrations de poissons vers les zones inondables du lac pendant la période de reproduction.

Cette région est caractérisée par un climat de transition entre le climat sub- équatorial de la côte et le climat tropical humide du type soudano-guinéen du Nord Bénin. Selon Adam et Boko (1993), ce climat est caractérisé par une grande saison pluvieuse (mi-mars à mi-juillet), une petite saison sèche (août), une petite saison pluvieuse (début septembre à mi-novembre) et une grande saison sèche (mi-novembre à mi-mars). Le lac connaît une période de basses eaux (novembre-mai) et une période de hautes eaux (juillet-septembre).

La végétation est constituée par une prairie aquatique flottante formant une auréole autour du lac Hlan. Elle est formée d'un enchevêtrement d'Eichinochloa pyramidalis, Brachiaria mutica, Ludwigia repens, Cyclosorus striatus dont le débordement est fait de Nymphaea lotus, Pistia stratiotes, Ipomoea aquatica, Salvinia nymphellula. Il est parfois constitué exclusivement d'Eichinochloa pyramidalis et/ou de Brachiaria mutica. On y rencontre d'autres

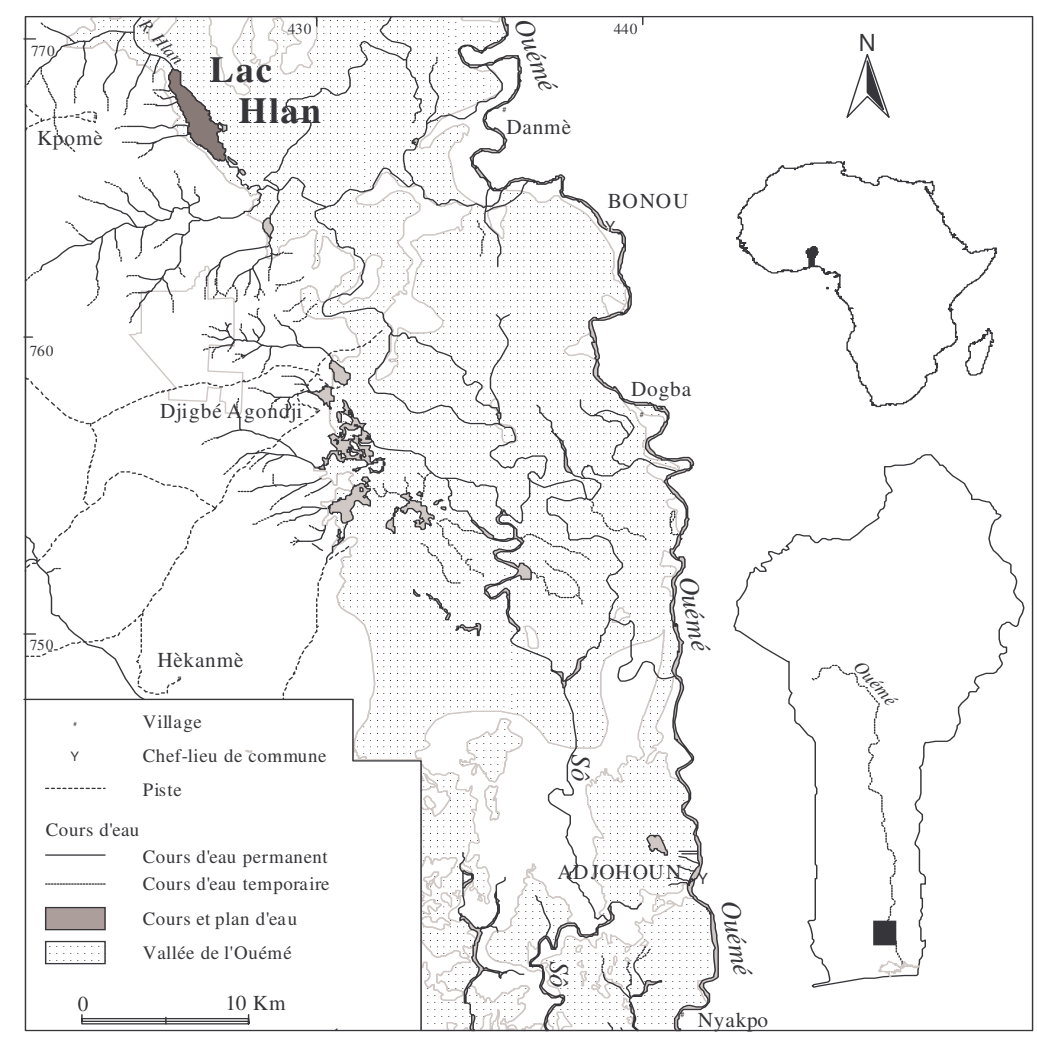

Figure 1: Localisation du lac Hlan. 
espèces telles que Lemna paucicostata, Azolla africana, Eichhornia crassipes, Ceratophyllum demersum, Neptunia oleracea. Ce tapis vert est par endroit découpé soit de manière à rendre les abords plus ou moins rectilignes le long desquels sont posés les filets maillants soit en cordons disposés en cercle dont les filets sont à la fois posés aux abords intérieur et extérieur de ce dernier. Cette dernière constitue une technique locale de pêche appelée « houéko »; ce qui signifie une "végétation flottante à poissons". Celle-ci représente une importante source de nourriture et constitue des frayères pour plusieurs espèces de poissons.

\section{Echantillonnage des Poissons}

La collecte des données sur le terrain a duré 12 mois (septembre 2004 à août 2005). Les échantillons mensuels de poissons sont principalement issus des captures de la pêche artisanale. Celle-ci utilise toute une panoplie d'engins et de techniques de pêche dont les filets maillants (maille étirée comprise entre 10 et $100 \mathrm{~mm}$ ), les nasses, les lignes simples et composées (palangres) appâtées et les filets de barrage. Les données d'inventaire de la pêche artisanale sont complétées par celles des pêches expérimentales avec la senne à très fine maille (filet moustiquaire) utilisée dans les zones peu profondes. La senne moustiquaire a été utilisée dans le but d'échantillonner des espèces de petites tailles qui auraient échappé à la pêche conventionnelle. Chaque espèce de poisson est identifiée suivant les guides d'identification taxonomiques des poissons de l'Afrique de l'Ouest (Levêque et al., 1990, 1992). Les échantillons de poissons sont mesurés et pesés respectivement avec un ichtyomètre (au mm près) et une balance électronique KERN (précision 0,1g).

\section{Analyse des données}

Après avoir dressé la liste des différentes espèces recensées au cours de cette étude, une analyse comparative a été faite par rapport aux études antérieures (Lalèyè et al., 1997). Pour une présentation synthétique et une meilleure compréhension de l'organisation des peuplements de poissons du lac Hlan, nous avons calculé l'indice H' de diversité de Shannon et Weaver (1948) et la régularité ou l'équitabilité E de Piélou (1969).
Ces deux indices sont calculés suivant les formules suivantes :

$\mathrm{H}^{\prime}=-\sum \mathrm{p}_{\mathrm{i}} \log _{2} \mathrm{p}_{\mathrm{i}}$ avec $\mathrm{p}_{\mathrm{i}}=\mathrm{ni} / \mathrm{N}$ où $\mathrm{N}$ est la somme des effectifs des espèces; ni, l'effectif de la population liée à chaque espèce; $p_{i}$, l'abondance relative de l'espèce i dans l'échantillon.

$\mathrm{E}=\mathrm{H}^{\prime} / \log _{2} \mathrm{~S}$. $\mathrm{S}$ est la richesse spécifique c'est-à-dire le nombre d'espèces constituant le peuplement. Cet indice permet de savoir si les individus sont équitablement répartis au sein du peuplement. Ces indices sont calculés mensuellement pour apprécier leur variation dans le temps.

Pour caractériser la structure en tailles de la communauté des poissons du lac Hlan, on a établi la distribution des fréquences de tailles de l'ensemble des individus pêchés. Cela a permis de déterminer les tailles dominantes dans les captures. Les abondances numériques (densité) et pondérales (biomasse) ont été également calculées mensuellement pour la communauté.

\section{Traitements statistiques}

Les variations mensuelles de richesse spécifique, de densité et des indices de Shannon (H') et de Piélou (E) sont comparées au moyen du test Kruskal-Wallis. La variation mensuelle de biomasse est comparée au moyen du test d'ANOVA. Une Analyse Factorielle des Correspondances (AFC) a été réalisée à partir du groupe responsable de $80 \%$ des abondances numérique et pondérale (une dizaine d'espèces) afin d'avoir une idée de l'organisation de l'assemblage. Les données brutes sur l'ensemble des espèces ne permettent pas une analyse statistique correcte car certaines d'entre elles sont peu représentées dans le temps. Les espèces occasionnelles ont donc été éliminées. Pour une standardisation des variables, nous avons utilisé une transformation des valeurs de ces abondances en prenant le $\ln (\mathrm{x}+1)$ (Legendre et Legendre, 1979). Les résultats de l'AFC ont été complétés par une classification hiérarchique ascendante $(\mathrm{CAH})$ réalisée sur les axes de l'AFC qui extraient plus de 50\% de l'inertie totale. La CAH utilise la distance (1-r) de Pearson et la méthode d'agrégation de Ward. Cette méthode est recommandée par Lebart et al. (1997) pour faciliter les regroupements des variables sur les plans factoriels. Toutes les analyses statistiques ont 
été réalisées avec le logiciel Statistica (Version 7.1)

\section{RÉSULTATS}

\section{Richesse spécifique}

Le nombre total d'espèces identifiées au lac Hlan est de 37 (Tableau 1) réparties en 18 familles et 29 genres. Les familles les plus représentées sont les cichlidés (8 espèces), les mormyridés (4 espèces) et les cyprinidés (3 espèces). Ensuite, viennent les polypteridés, notopteridés, characidés, claroteidés, aplocheilidés, channidés avec chacune deux espèces. Les autres familles sont représentées chacune par une seule espèce.

La différence mensuelle de la richesse spécifique entre les mois (Figure 2) est significative $(\mathrm{p}<0,05)$. Par contre, cette différence n'est pas significative $(p>0,05)$ pour les indices de diversité de Shannon et Weaver (H') et de l'équitabilité de Pielou (E).

Les nombres les plus élevés d'espèces ( $n=19)$ sont observés pendant les mois de juin à octobre. Cette période correspond à la saison des crues. Par contre, de faible nombre d'espèces sont observés de novembre à mai pendant la décrue.

Les indices $H^{\prime}$ et $\mathrm{E}$ présentent également leurs valeurs maximales en septembre et en octobre et les minimales en août et décembre (Figure 3). Cela montre qu'en dehors des crues, peu d'espèces représentent la majorité des captures.

\section{Abondances numérique (densité) et pondérale (biomasse) \\ Un effectif total de 1700 individus,} représentant une biomasse de 443,202 kg, a été capturé sur l'ensemble du lac Hlan pendant la période d'étude. On observe une évolution en dents de scie de la densité et de la biomasse (Figure 4). La différence mensuelle de densité et de biomasse entre les mois d'étude présente une différence significative $(\mathrm{p}<0,05)$. Les pics de densité et de biomasse ont été observés aux mois de novembre (fin de la crue) et de juin (début de la crue au niveau du lac).

Les cichlidés sont prépondérants en terme d'abondance numérique avec $65,5 \%$. Viennent ensuite et dans l'ordre les osteoglossidés $(6,9 \%)$, les claroteidés $(5,5 \%)$, les channidés $(3,6 \%)$, les clariidés $(2,6)$ et les gymnarchidés (1\%). En terme de biomasse, les osteoglossidés sont les premiers avec $44,9 \%$. Suivent dans l'ordre les cichlidés $(38,9 \%)$, les gymnarchidés $(6,4 \%)$, les clariidés $(3,2 \%)$, les channidés $(2,7 \%)$ et les claroteidés $(2,2 \%)$. La pression de pêche s'exerce essentiellement sur environ 10 espèces (Parachanna obscura, Gymnarchus niloticus, Heterotis niloticus, Hepsetus odoe, Clarias gariepinus, Chrysichthys auratus, Hemichromis fasciatus, Tilapia mariae, Tilapia zillii, Sarotherodon galilaeus) qui représentent plus de $80 \%$ de la densité et $95 \%$ de la biomasse.

\section{Structure de la communauté Structure en tailles}

L'analyse des structures en tailles des échantillons (Figure 5) montre que les poissons capturés sont généralement de grandes tailles. Plus de $80 \%$ ont une taille supérieure ou égale à $20 \mathrm{~cm}$ (en majorité des cichlidés). La plus petite taille observée dans les captures est inférieure à $10 \mathrm{~cm}$ (les cichlidés) et la plus grande taille observée est de 133,5 cm (Gymnarchus niloticus). Les petites tailles observées l'ont été pendant les mois de juillet, août et septembre ; période de reproduction pour la majorité des espèces où les juvéniles sont les plus nombreux.

\section{Caractéristiques des assemblages}

L'analyse du premier plan factoriel (axes I et II, 55,54\% de l'inertie totale) permet d'isoler 3 ensembles (Figure 6) : un premier groupe représenté par Sarotherodon galilaeus, Tilapia zillii et Tilapia mariae, un deuxième groupe constitué par Gymnarchus niloticus, Parachanna obscura et Heterotis niloticus et un troisième groupe composé de Hemichromis fasciatus, Chrysichthys auratus, Hepsetus odoe et Clarias gariepinus. Ce résultat est confirmé par l'analyse hiérarchique ascendante $(\mathrm{CAH})$ effectuée à partir des coordonnées des 7 premiers axes de l'AFC (Figure 7).

Le premier groupe de poissons est celui des espèces les plus représentées dans les captures commerciales et rencontrées presque tous les mois. Ce groupe, composé uniquement de cichlidés, est caractérisé par des espèces phytoplanctonophages. Il possède les plus fortes densités $(65,5 \%)$. Le deuxième groupe est composé par des espèces piscivores 
Tableau 1: Liste des espèces recensées dans le lac Hlan de septembre 2004 à août 2005.

\begin{tabular}{|c|c|}
\hline Familles & Espèces \\
\hline Protopteridae & Protopterus annectens (Owen, 1839) \\
\hline \multirow{2}{*}{ Polypteridae } & Polypterus senegalus Cuvier, 1829 \\
\hline & Erpetoichthys calabaricus Smith, 1866 \\
\hline Osteoglossidae & Heterotis niloticus (Cuvier, 1829) \\
\hline \multirow{2}{*}{ Notopteridae } & Xenomystus nigri (Günther, 1868) \\
\hline & Papyrocranus afer (Günther, 1868) \\
\hline \multirow{4}{*}{ Mormyridae } & Mormyrus rume valenciennes, 1846 \\
\hline & Hyperopisus bebe (Lacépède, 1803) \\
\hline & Marcusenius senegalensis (Steindachner, 1870) \\
\hline & Brevimyrus niger (Günther, 1866) \\
\hline Gymnarchidae & Gymnarchus niloticus Cuvier, 1829 \\
\hline Hepsetidae & Hepsetus odoe (Bloch, 1794) \\
\hline \multirow{2}{*}{ Characidae } & Brycinus longipinnis (Günther, 1864) \\
\hline & Brycinus nurse (Rüppel, 1832) \\
\hline Distichodontidae & Distichodus rostratus Günther, 1864 \\
\hline \multirow{3}{*}{ Cyprinidae } & Barbus callipterus Boulenger 1907 \\
\hline & Barbus leonensis Boulenger, 1915 \\
\hline & Prolabeo batesi Norman, 1932 \\
\hline \multirow{2}{*}{ Claroteidae } & Chrysichthys nigrodigitatus (Lacépède, 1803) \\
\hline & Chrysichthys auratus (Geoffroy Saint-Hilaire, 1808) \\
\hline Schilbeidae & Schilbe intermedius Rüppel, 1832 \\
\hline \multirow{2}{*}{ Clariidae } & Clarias gariepinus (Burchell, 1832) \\
\hline & Clarias ebriensis Pellegrin, 1920 \\
\hline \multirow{2}{*}{ Aplocheilidae } & Epiplatys grahami (Boulenger, 1911) \\
\hline & Aphyosemion guineense Daget, 1954 \\
\hline \multirow{2}{*}{ Channidae } & Parachanna africana (Steindachner, 1879) \\
\hline & Parachanna obscura (Günther, 1861) \\
\hline Nandidae & Polycentropsis abbreviata Boulenger 1901 \\
\hline \multirow{8}{*}{ Cichlidae } & Chromidotilapia guntheri (Sauvage 1882) \\
\hline & Hemichromis fasciatus Peters, 1852 \\
\hline & Hemichromis bimaculatus Gill, 1862 \\
\hline & Tilapia mariae Boulenger, 1899 \\
\hline & Tilapia zillii (Gervais, 1848) \\
\hline & Oreochromis niloticus (Linné, 1758) \\
\hline & Sarotherodon melanotheron Rüppel, 1852 \\
\hline & Sarotherodon galilaeus (Linné, 1758) \\
\hline Anabantidae & Ctenopoma petherici Günther, 1864 \\
\hline
\end{tabular}

à l'exception de Heterotis niloticus. Cet assemblage possède les plus fortes biomasses (54\%) et est caractérisé par des individus de grande taille. Le troisième groupe comporte des espèces qui sont peu représentées dans les captures. Ce groupe de poissons est celui des espèces qui complètent les espèces des deux premiers groupes dans les captures artisanales commerciales. Il est caractérisé par un assemblage de piscivores (Hepsetus odoe et Hemichromis fasciatus) et d'omnivores (Clarias gariepinus et Chrysichthys auratus). 


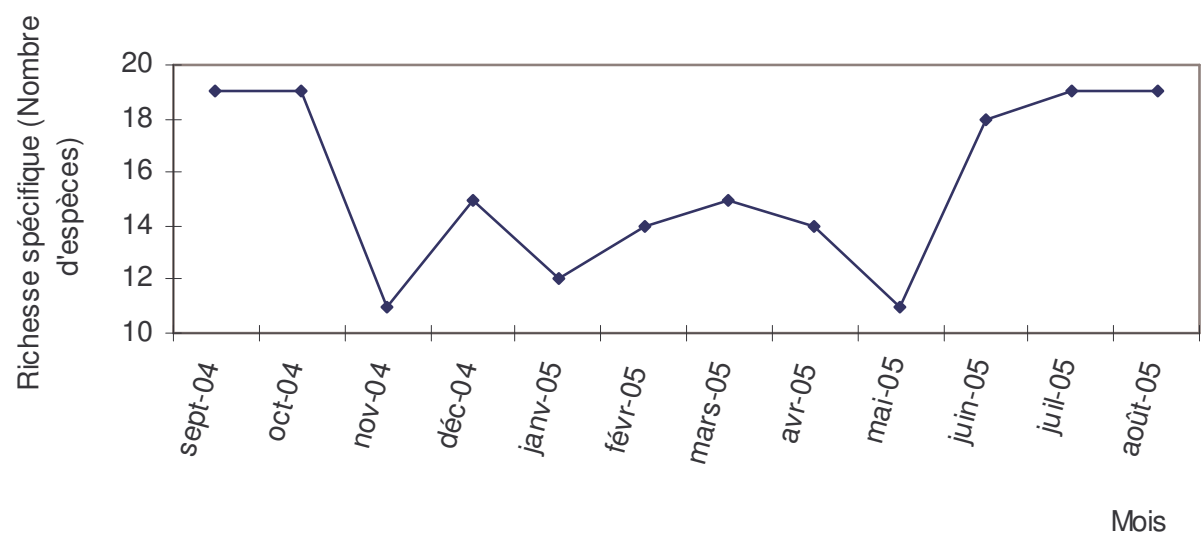

Figure 2: Evolution mensuelle de la richesse spécifique du lac Hlan (de septembre 2004 à août 2005).

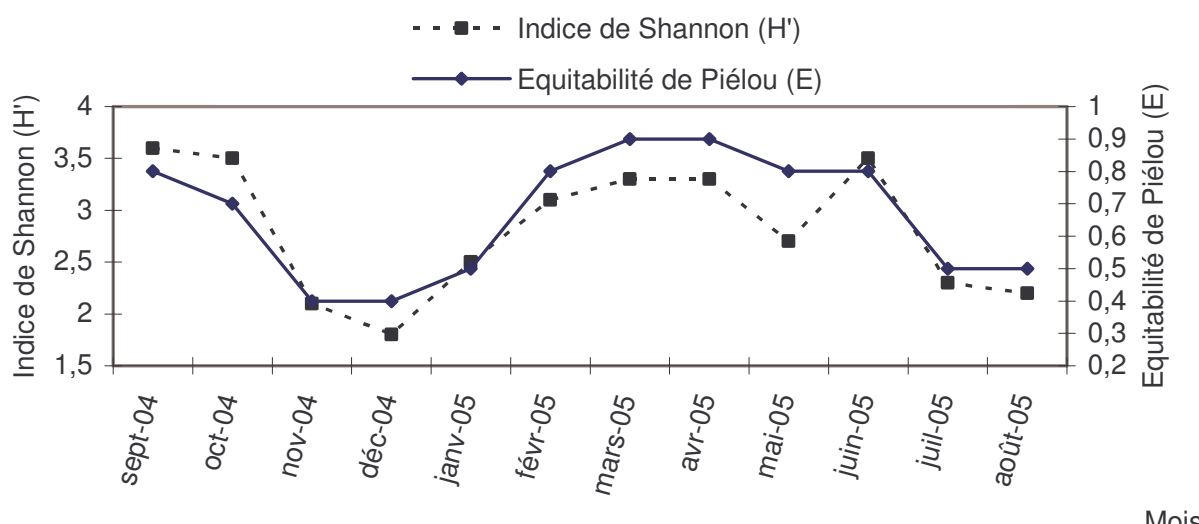

Figure 3: Evolution mensuelle de l'Indice de Shannon (H') et de l'Equitabilité de Piélou (E) de septembre 2004 à août 2005 dans le lac Hlan.

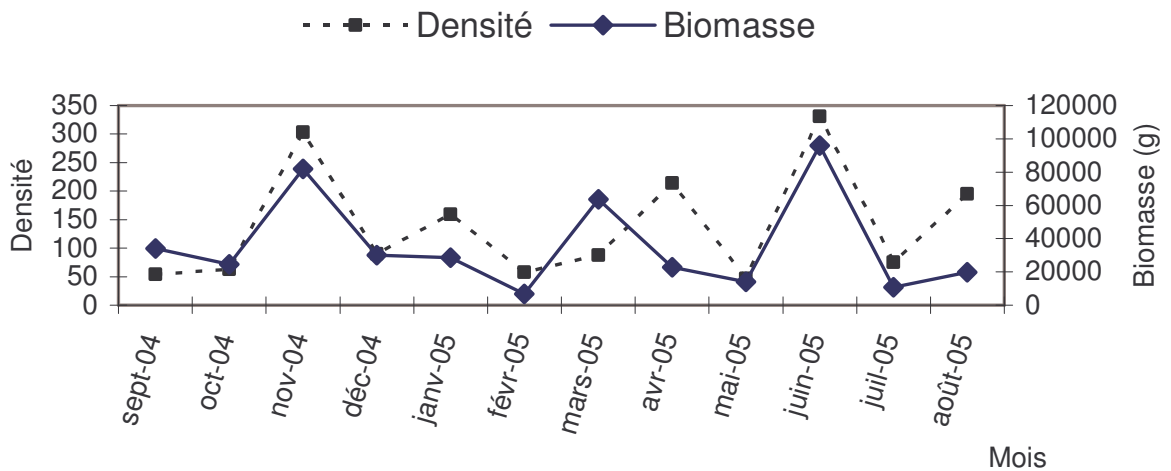

Figure 4: Evolution mensuelle de la densité et de la biomasse des poissons du lac Hlan de septembre 2004 à août 2005. 


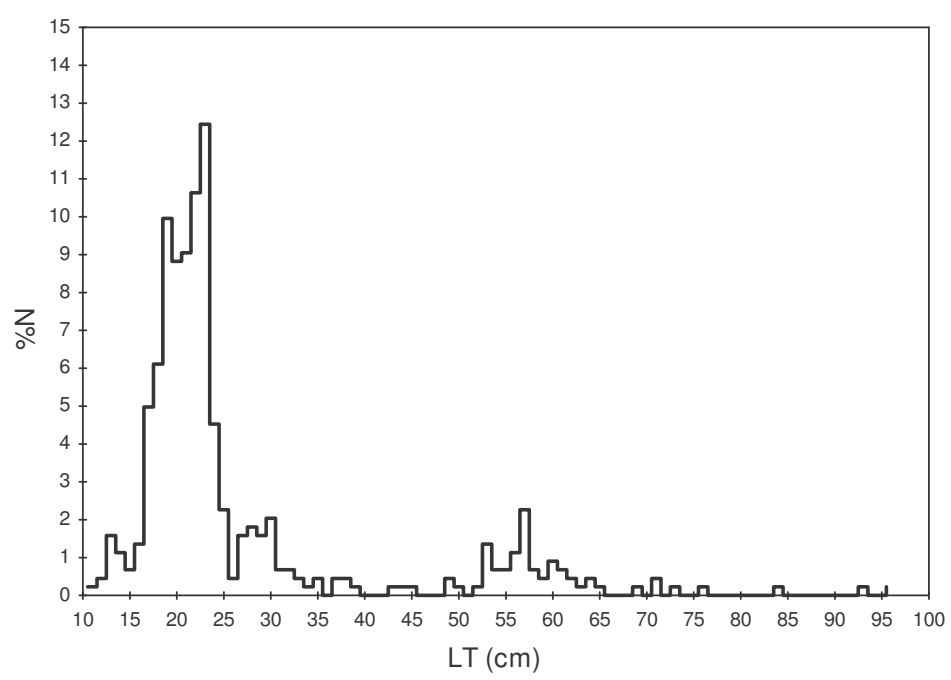

Figure 5: Structure générale en tailles des poissons collectés dans le lac Hlan de septembre 2004 à août 2005.

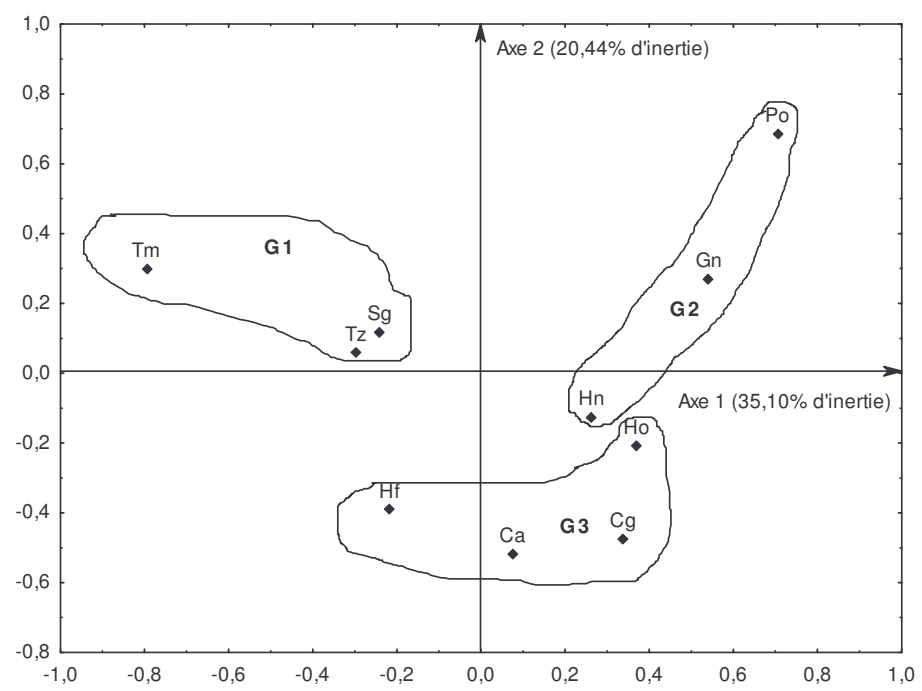

Figure 6: Analyse factorielle des correspondances sur le groupe responsable de $80 \%$ des abondances dans le lac Hlan. Po: Parachanna obscura, Gn: Gymnarchus niloticus, Hn: Heterotis niloticus, Ho: Hepsetus odoe, Cg: Clarias gariepinus, Ca: Chrysichthys auratus, Hf: Hemichromis fasciatus, Tm: Tilapia mariae, Tz: Tilapia zillii, Sg: Sarotherodon galilaeus.

\section{DISCUSSION}

\section{Richesse spécifique}

Le lac Hlan comporte une faune ichtyologique assez diversifiée qui reflète celle de la rivière Hlan (Montchowui et al., 2007) et celle du fleuve Ouémé (Lalèyè et al., 2004) auxquelles elle est associée. Le lac Hlan est un plan d'eau de type ouvert (Leveque, 1999) et est en communication permanente avec la rivière Hlan qui est son principal bassin versant. Il est aussi en communication temporaire avec le fleuve Ouémé et la rivière Sô pendant les crues. La comparaison de la faune ichtyologique avec la situation antérieure est difficile car il n'existe pas dans la littérature d'information précise sur la faune de ce lac. Seuls Lalèyè et al. (1997) ont établi une liste des espèces du lac Hlan à partir d'observations ponctuelles effectuées sur le terrain. Les taxons dont ces auteurs ont 


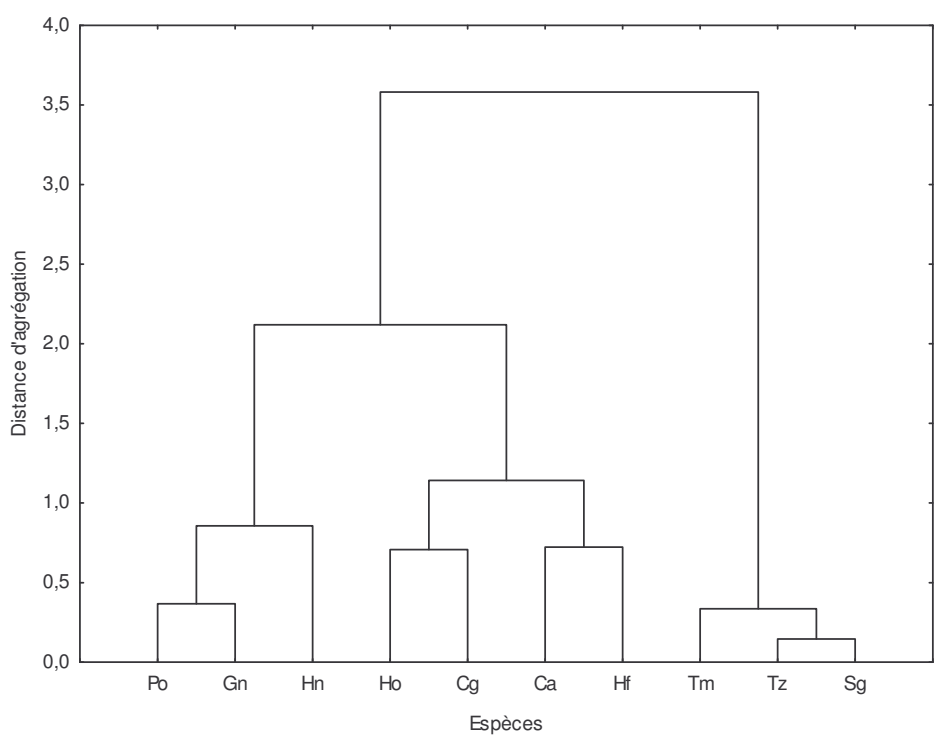

Figure 7: Analyse hiérarchique ascendante sur le groupe responsable de $80 \%$ des abondances dans le lac Hlan. Po: Parachanna obscura, Gn: Gymnarchus niloticus, Hn: Heterotis niloticus, Ho: Hepsetus odoe, Cg: Clarias gariepinus, Ca: Chrysichthys auratus, Hf: Hemichromis fasciatus, Tm: Tilapia mariae, Tz: Tilapia zillii, Sg: Sarotherodon galilaeus.

signalé la disparition ou la rareté, sont présents dans notre liste à l'exception de Clarias agboyiensis. Il s'agit de Gymnarchus niloticus (la plus citée) et de Parachanna africana.

La composition de l'icthyofaune du lac Hlan ressemble à celle observée dans des lacs africains de même type tels que Ihéma (Rwanda), Kainji (Nigéria), Kangimi (Nigéria), Tiga (Nigéria), Georges (Uganda) et Toho-todougba (Bénin) où les cichlidés constituent les composantes importantes de l'ichtyofaune (Gwahaba, 1975; Ita, 1982; Balogun, 1986; Plisnier et al., 1988 ; Adite et Winemiller, 1997; Balogun, 2005).

La variation temporelle de la richesse spécifique observée sur le lac Hlan n'est pas un phénomène nouveau dans les milieux lacustres de type ouvert (Leveque, 1999). Par définition, des variations temporelles des assemblages peuvent concerner le nombre d'espèces (Thayer et al., 1987), ainsi que leur abondance relative (Albaret et Ecoutin, 1990 ; Aliaume et al., 1993). Les variations sont liées soit aux changements comportementaux des individus, qui deviennent plus ou moins vulnérables aux engins de pêche, soit aux migrations des populations (Bouchereau et al., 1991; Bouchereau, 1997). Dans le lac Hlan, les variations mensuelles de la richesse spécifique sont beaucoup plus liées aux migrations des espèces entre le lac et ses rivières associées pendant les périodes de faibles et hautes eaux. En effet, la richesse spécifique la plus élevée est observée pendant les mois de juin, juillet, août, septembre et octobre qui correspondent à la saison des crues sur le lac Hlan. Les poissons migrent de l'aval de la rivière Sô et du fleuve Ouémé vers le lac Hlan et sa plaine d'inondation à la recherche de sites propices pour frayer. En revanche, de faibles richesses spécifiques sont observées de novembre à mai. C'est la période de décrue et les espèces qui sont venues se reproduire dans les zones marginales peu profondes du lac migrent dans le sens inverse. Le phénomène de migration de populations de poissons entre le lac Hlan et les cours d'eau associés a été signalé par Adite et al. (2005, 2006). Ces auteurs rapportent la migration de Heterotis niloticus entre le lac Hlan, la rivière Sô et les plaines d'inondation associées pendant les hautes eaux et à la décrue.

Cette hypothèse est corroborée par les variations mensuelles de la densité et de la biomasse des poissons pêchés par la pêche artisanale. En effet, les pics de densité et de biomasse de l'ichtyofaune (Figure 4) ont été 
observés au début (juin) et à la fin (novembre) de la période des crues au niveau du lac; ce qui montre que beaucoup d'espèces arrivent en début de crues au niveau du lac et retournent une fois que le niveau de l'eau commence à diminuer.

Les cichlidés sont les plus nombreux dans le lac et dominent numériquement dans les captures artisanales. Contrairement à la dominance de Sarotherodon galilaeus et de Oreochromis niloticus dans les lacs Kainji, Tiga et Bakolori au Nigéria et des Hemichromis fasciatus dans le lac Kangimi (Nigéria) (Balogun, 2005), ce sont Tilapia zillii et Sarotherodon galilaeus qui dominent au lac Hlan. Ce résultat est similaire à celui rapporté par Paugy (1979) dans les lacs de barrage de Côte d'Ivoire où Sarotherodon galilaeus et Tilapia zillii constituaient respectivement $42,3 \%$ et $13,9 \%$ des captures.

En terme d'abondance pondérale, c'est Heterotis niloticus qui domine dans les captures de la pêche artisanale au lac Hlan avec près de $44,4 \%$. Suivent Sarotherodon galilaeus et Tilapia zillii avec environ $18 \%$ chacune. Ces trois espèces participent de façon significative (environ 80\%) aux abondances des captures de la pêche artisanale toute l'année et dominent le peuplement. Les autres espèces participant aux captures commerciales de la pêche artisanale complètent cet éventail assez réduit. Un résultat similaire a été obtenu par Baijot et al. (1994) dans les petits lacs de barrage de Burkina-Faso où 3 espèces constituaient à eux seuls plus de $90 \%$ des captures. Il s'agit de Clarias gariepinus (fortement dominant), Oreochromis niloticus et Sarotherodon galilaeus.

\section{Structure et caractéristiques des assemblages}

L'étude de la structure des communautés de poissons a permis d'isoler trois groupes de poissons dans le lac Hlan. Le premier groupe est composé des espèces présentes dans le lac toute l'année. Ce sont en majorité des espèces de cichlidés qui participent de façon significative aux captures commerciales. Elles pourraient être considérées comme des espèces résidentes permanentes (Elliot and Dewailly, 1995). Le second groupe est composé exclusivement d'espèces de grande taille. Elles sont capturées pour la majorité des cas pendant les crues, période de la reproduction chez la majorité des poissons du lac Hlan où les larves et juvéniles issus de la reproduction de l'année sont les plus nombreuses ; conditions alimentaires favorables pour les espèces piscivores. Le troisième groupe est celui des espèces complétant celles des deux premiers groupes dans les captures de la pêche artisanale.

Les trois groupes de poissons identifiés par l'AFC et la CAH peuvent être répartis en trois guildes alimentaires: détritivores, omnivores et piscivores (Lauzanne, 1976; Hugueny and Pouilly, 1999). Les espèces détritivores sont Sarotherodon galilaeus, Tilapia zillii et Tilapia mariae tandis que les omnivores sont représentées par Heterotis niloticus, Clarias gariepinus et Chrysichthys auratus. Les espèces piscivores sont Hemichromis fasciatus, Hepsetus odoe, Parachanna obscura et Gymnarchus niloticus.

$\mathrm{La}$ forte concentration des espèces détritivores au niveau du lac indique que ce milieu offre des conditions écologiques (ressources alimentaires et habitats de reproduction) permettant l'établissement de ces espèces; ceci favoriserait un bon recrutement du lac assurant le renforcement continuel du stock de ces espèces qui $\mathrm{y}$ demeurent tout au long de l'année. En effet, le lac Hlan dispose de différents types d'habitats qui pourraient offrir une disponibilité en ressources alimentaires due à la présence de végétation flottante, herbacée et à l'émergence et la submergence de la végétation terrestre adjacente. Ces habitats constitueraient également des frayères propices pour ces espèces et favoriseraient une importante colonisation par les poissons.

La majorité des espèces des trois groupes de poissons formant l'assemblage au lac Hlan sont des espèces ubiquistes (Bouchereau et al., 2000) occupant tous les endroits du lac où la nourriture est disponible. Elles sont pour la plupart des consommateurs primaires et secondaires. La dominance des consommateurs primaires et secondaires au lac Hlan indique un fort potentiel de production de poissons.

En effet, les poissons capturés au moyen des différents engins de pêche artisanale dans le lac sont généralement de grandes tailles. Plus de $80 \%$ ont une taille 
supérieure ou égale à $20 \mathrm{~cm}$ (en majorité des cichlidés) avec des maxima allant jusqu'à 95 $\mathrm{cm}$ en moyenne (Heterotis niloticus et Gymnarchus niloticus). Cette situation inhabituelle et contraire à ce qu'on observe dans la plupart des écosystèmes aquatiques du sud-Bénin où les captures sont constituées des poissons de petites tailles (Lalèyè et al., 2003) est due à une réglementation traditionnelle locale de gestion. L'utilisation par exemple du filet épervier, qui capture les individus de toute taille, est interdite par la réglementation locale. Le stock halieutique de ce plan d'eau est relativement moins dégradé que dans la plupart des autres écosystèmes aquatiques du sud-Bénin. De gros spécimens de Gymnarchus niloticus, ménacés dans la plupart des plans et cours d'eau du Bénin, sont encore capturés au lac Hlan. Cette gestion traditionnelle doit être appuyée et renforcée pour une exploitation durable des stocks de poissons de ce lac.

\section{REMERCIEMENTS}

Les auteurs remercient sincèrement l'INRAB (Institut National des Recherches Agricoles du Bénin) et l'ABE (Agence Béninoise pour l'Environnement) qui ont soutenu financièrement ce travail. Nous remercions également les pêcheurs qui ont aidé à la collecte des données sur le terrain et les collègues du Laboratoire d'Hydrobiologie et d'Aquaculture de l'Université d'AbomeyCalavi (Bénin) pour leur contribution. Nous remercions également les lecteurs anonymes qui ont porté des corrections et critiques constructives à ce manuscrit.

\section{RÉFÉRENCES BIBLIOGRAPHIQUES}

Adam KS, Boko M. 1993. Le Bénin. Edicef: Paris; 96 p.

Adité A, Rudi VT. 1995. Ecology and Fish catches in natural lakes in Benin, West Africa. Environ. Biol Fishes., 43: 381391.

Adité A, Winemiller KO. 1997. Trophic ecology and ecomorphology of fish assemblages in coastal lakes of Benin, West Africa. Ecoscience, 4: 6-23.

Adité A, Winemiller KO, Fiogbe ED. 2005. Ontogenic, seasonal, and spatial variation in diet of Heterotis niloticus (Osteoglossiformes: Osteoglosidae) in Sô
River and Lake Hlan, Benin, West Africa. Environ. Biol Fishes., 73: 367-378.

Adité A, Winemiller KO, Fiogbe ED. 2006. Population structure and reproduction of the African bonytongue Heterotis niloticus in the Sô River-floodplain system (West Africa): implications for management. Ecol. Fresh Fish., 15: 3039.

Albaret JJ, Ecoutin JM. 1990. Influence des saisons et des variations climatiques sur les peuplements de poissons d'une lagune tropicale en Afrique de l'Ouest. Acta Oecologia, 11: 557-583.

Aliaume C, Monteiro C, Louis M, Lam Hoai T, Lasserre G. 1993. Organisation spatiotemporelle des peuplements ichtyologiques dans deux lagunes côtières : au Portugal et en Guadeloupe. Oceanologica Acta, 16: $291-301$

Baijot E, Barry I, Ratjs F. 1994. Peuplements piscicoles des retenues du Burkina Faso. In Aspects Hydrobiologiques et Piscicoles des Retenues d'Eau en Zone SoudanoSahélienne. Le Cas du Burkina Faso, Baijot E, Moreau J, Bouda S (eds). Centre Technique de Coopération Agricole et Rurale: Van Ruys-Bruxelles; 65-85.

Balogun JK. 1986. Fish distribution in Kainji Lake, Nigeria. J. Fish. Biol., 29: 489498.

Balogun JK. 2005. Fish distribution in a small domestic water supply reservoir: A case study of Kangimi reservoirs, Kaduna Nigeria. J. Appl. Sci. Environ. Mgt., 9: 93-97.

Bouchereau JL. 1997. Biodiversity of tactics used by three Gobiidae (Pisces, Teleostei): Pomatoschistus minutus (Pallas, 1770), P. microps (Krøyer, 1838), Gobius niger Linnæus, 1758, to survive in a Mediterranean lagoon environment. Oceanological Studies, 2-3: 153-170.

Bouchereau JL, Durel JS, Guelorget O, ReyNau Louali L. 2000. L'ichtyofaune dans l'organisation biologique d'un système paralique marocain : la lagune de Nador. Mar. Life., 10: 69-76.

Bouchereau JL, Quignard JP, Joyeux JC, Tomasini JA. 1991. Stratégies et tactiques de reproduction de Pomatoschistus microps (Krøyer, 1838) et Pomatoschistus minutus (Pallas, 1770) (Pisces, Gobiidae) dans le Golfe du Lion 
(France), Nids, déterminisme de la sédentarité et de la migration. Cybium, 15: 315-345.

Elliot M, Dewailly F. 1995. The structure and components of European estuarine fish assemblages. Neth. J. Aquat. Ecol., 29: 397-417.

Gwahaba JJ. 1975. The distribution, population density and biomass of fish in an equatorial lake, Lake Georges, Uganda. Proc. R. Soc., Lond., 190: 393414.

Hugueny B, Pouilly M. 1999. Morphological correlates of diet in an assemblage of West Africa freshwater fishes. J. Fish Biol., 54: 1310-1325.

Ita EO. 1982. Fisheries survey of Tiga Lake, Kano State, Nigeria. Kainji. Lake Research Report: 67-74.

Lalèyè P. 1995. Ecologie comparée de deux espèces de Chrysischthys, Poissons siluriformes (Claroteidae) du complexe lagunaire lac Nokoué - lagune de PortoNovo au Bénin. Thèse de doctorat, Université de Liège, Liège, p.152.

Lalèyè P, Chikou A, Wuemènou T. 1997. Poissons d'eaux douces et saumâtres du Bénin : Inventaire, distribution, statut et conservation. Inventaire des poissons menacés de disparition du Bénin (Rapport d'études). Coop. Bénino-néerlandaise/ Ambassade Royale des Pays-Bas, Cotonou, Bénin, 97p.

Lalèyè $\mathrm{P}$, Niyonkuru $\mathrm{C}$, Moreau $\mathrm{J}$, Teugels GG. 2003. Spatial and seasonal distribution of the ichtyofauna of Lake Nokoué, Bénin, West Africa. Afr. J. of Aqu. Sc., 28: 151-161.

Lalèyè $\mathrm{P}$, Chikou A, Philippart JC, Teugels GG, Vandewalle P. 2004. Etude la diversité ichtyologique du bassin du fleuve Ouémé au Bénin (Afrique de l'Ouest). Cybium, 28: 329-339.

Lauzanne L. 1976. Régimes alimentaires et relations trophiques des poissons du lac Tchad. Cah. ORSTOM, ser. Hydrobiol., 4: 267-310.

Lebart L, Morineau A, Piron M. 1997. Statistique Exploratoire Multidimensionnelle ( $2^{\text {nde }}$ édn). Dunod Publications: Paris; 439p.
Legendre L, Legendre P. 1979. Ecologie Numérique 2. Structure des Données Ecologiques. Masson et Presses Universitaires: Québec ; 254p.

Levêque C. 1999. Les peuplements des lacs peu profonds. In Les Poissons des Eaux Continentales Africaines. Diversité, Écologie, Utilisation par l'Homme, Levêque C, Paugy D (eds). Institut de Recherche pour le Développement: Paris ; 311-323.

Levêque C, Paugy D, Teugels GG. 1990. Faune des Poissons d'Eaux Douces et Saumâtres de l'Afrique de l'Ouest. Tome 1. Collection Faune Tropicale, $\mathrm{n}^{\circ}$ XXVIII. ORSTOM/MRAC: Paris.

Levêque C, Paugy D, Teugels GG. 1992. Faune des Poissons d'Eaux Douces et Saumâtres de l'Afrique de l'Ouest. Tome 2. Collection Faune Tropicale, $n^{\circ}$ XXVIII. ORSTOM/MRAC: Paris.

Levêque C, Paugy D. 1999. Impacts des activités humaines. In Les Poissons des Eaux Continentales Africaines. Diversité, Ecologie, Utilisation par l'Homme, Levêque C, Paugy D (eds). Institut de Recherche pour le Développement: Paris; 365-383.

Montchowui E, Niyonkuru C, AhouansouMontcho S, Chikou A, Lalèyè P. 2007. L'ichtyofaune de la rivière Hlan au Bénin (Afrique de l'Ouest). Cybium, 31: 173176.

Paugy D. 1979. Les Peuplements Ichtyologiques des Lacs de Barrage de Côte d'Ivoire. ORSTOM: Paris, Mimeo.

Pielou EC. 1969. An Introduction to Mathematical Ecology. John Wiley \& Sons: New York.

Plisnier PD, Micha JC, Frank V. 1988. Biologie et Exploitation des Poisons du Lac Ihema (Bassin Akagera, Rwanda). Presses Universitaires de Namur: Belgique.

Shannon CE, Weaver W. 1948. The Mathematical Theory of Communication. Univ. Illinois Press: Urbana, Illinois; $117 \mathrm{p}$.

Thayer GW, Colby DR, Hettler WFJ. 1987. Utilization of the red mangrove proper root habitat by fishes in south Florida. Mar. Ecol. Prog. Ser, 35: 25-38. 